\title{
Mehrebenen-Ansätze - Komplexe Projektarchitekturen in der Organisationsentwicklung
}

\author{
S. Kunert • S. Bedenk
}

Eingegangen: 10. Februar 2021 / Angenommen: 15. März 2021 / Online publiziert: 16. April 2021

(C) Der/die Autor(en) 2021

Zusammenfassung Dieser Artikel widmet sich beraterischen Prinzipien für die Konstruktion von komplexen Projektarchitekturen zur Organisationsentwicklung. Anhand der Gestaltungsgebote Spezifität und Symmetrie, Oszillation, Integration, Choreographie, Phasen, Kaskadierung, Agilität und Adaptivität sowie Anschlussfähigkeit und Irritation werden systemische Grundsätze der Prozessberatung auf die Planung, Supervision und Evaluation von größeren Veränderungsvorhaben übertragen. An einer Fallstudie wird die Anwendung dieser Prinzipien exemplarisch demonstriert.

Schlüsselwörter Mehrebenen-Architektur · Organisationsentwicklung · Systemische Prinzipien

Dr. S. Kunert $(\bowtie)$

Humboldt-Universität zu Berlin, Unter den Linden 6, 10117 Berlin, Deutschland

E-Mail: kunerseb@hu-berlin.de

Dr. S. Bedenk $(\bowtie)$

artop $\mathrm{GmbH}$ - Institut an der Humboldt-Universität zu Berlin, Christburger Str. 4, 10405 Berlin, Deutschland

E-Mail: bedenk@artop.de 


\section{Multi-level designs-complex project schedules in organisational development}

Abstract In this article, we postulate principles for building complex schedules for organisational development projects. In particular, this includes specificity and symmetry, oscillation, integration, choreography, stages, cascade, patterns, agility and adaptivity, connectivity and irritation. Finally, we show how to apply these systemic principles in a case study.

Keywords Multi-level schedule $\cdot$ Organizational development $\cdot$ Systemic principles

\section{Einleitung}

Die systemische Organisationsentwicklung hat ihren festen Platz in den Organisations- und Beratungswissenschaften. Selbst betriebswirtschaftlich und arbeitswissenschaftlich geprägte Quellen nehmen die Grundsätze und Vorgehensweisen der prozessbasierten personenzentrierten Veränderung in ihren Wissenskanon auf (eindrücklich bei Schanz 1994; Schlick et al. 2018). Dass systemische Beratung einen Mehrwert liefert, den sie von anderen professionellen Unterstützungsangeboten abhebt (Kunert und Bedenk 2013), wird kaum noch bezweifelt. Stattdessen rückt die Frage nach der Binnendifferenzierung bzw. Integration innerhalb der personenbezogenen Prozessberatung zusehends in den Mittelpunkt. Besonders in groß angelegten, teils mehrjährigen Veränderungsprojekten zeigt sich, wie mächtig eine abgestimmte Anwendung verschiedener systemischer Herangehensweisen sein kann (z. B. Heitger und Serfass 2015). Damit dies gelingt, braucht es allerdings einiger Vorkehrungen.

Projektarchitekturen sind zunächst nur ein Hilfsmittel zur Planung, entlehnt aus dem klassischen Projektmanagement. Zugleich spiegelt sich in ihnen die ganze Bandbreite an Grundannahmen über professionelle Organisationsentwicklung. ,Gute Architekturen von Beratungsprozessen erleichtern neue Sichtweisen, ermöglichen vielfältige Perspektiven (z. B. durch heterogene Gruppenzusammensetzungen), führen neue Unterschiede ein (z.B. durch Interviews mit Kunden) und eröffnen neue Beobachtungs- bzw. Reflexionsmöglichkeiten (z. B. durch Elemente wie das Soundingboard). Dadurch werden Unterbrechungen von Mustern erleichtert, Gesamtentwicklungen gefördert, das Lernenlernen und die Selbststeuerung beschleunigt" (Königswieser und Exner 2019, S. 49).

Systemischen Interventionen ist die Nicht-Steuerbarkeit ihrer Wirkung immanent. Dadurch wächst die Gefahr, ein Format- und Methodenfeuerwerk zu zünden in der Hoffnung, dass die beratene Institution von selbst für die nötige Integration der Veränderungsimpulse sorgt. Unserer Meinung nach gehört zu einer professionellen Prozessberatung nicht nur der kompetente Einsatz einzelner Formate, sondern auch die Anwendung systemischer Grundsätze auf der Meta-Ebene, also bei der Planung, Supervision und Evaluation von Veränderungsprojekten. In dem vorliegenden Artikel beleuchten wir Prinzipien von Projektarchitekturen zur Organisationsentwicklung im Sinne von Landkarten als ,soziale, zeitliche, räumliche und inhaltliche Gestal- 
tungselemente und Fixpunkte, die Prozesse vorstrukturieren“" (ebd., S. 47). Dabei integrieren wir verschiedene beraterische Interventionen als Formate (zur Herleitung des Format-Begriffs als professionelle Interaktion in der Organisationsentwicklung vgl. Kranz 2009; zur Anwendung vgl. v. Ameln und Kramer 2007, S. 5 ff.). In einem Fallbeispiel demonstrieren wir, wie dies praktisch umsetzbar ist.

\section{Komplexität in Organisationsentwicklungsprozessen}

Organisationen aller Art erleben einen nie gekannten Veränderungsdruck. So gut wie keine Branche bleibt von Digitalisierungs-, Markt-, Kunden- und Gesellschaftsveränderungen verschont. Diese Dynamik findet auch Einzug in die Organisationsentwicklung, wo die Anforderungen an institutionelle Wandelprozesse steigen. Dies erzeugt einerseits einen Professionalisierungsschub auf Kundenseite, wo Veränderungsmanagement längst zur Führungs- und HR-Aufgabe geworden ist und viele - zunehmend auch mittelständische und behördliche - Institutionen die OE-Funktion intern abdecken. Andererseits steigt der Bedarf nach integrierten, ganzheitlichen Lösungen, von denen man sich verspricht, mehrere Veränderungsbedarfe gebündelt zu bedienen, mehr Stakeholder-Gruppen simultan einzuspannen und nachhaltigere Lösungen zu generieren (vgl. Lippold 2020, S. 6).

Konzeptionell betrachtet sind Mehrebenen-Ansätze zunächst einmal eine Reaktion im Sinne des Ashbyschen Gesetzes (Ashby 1956): Der Komplexität in den Umweltanforderungen und damit in den organisationalen Veränderungsbedarfen begegnet man mit einer Gegenkomplexität im Changemanagement. Darüber hinaus trägt dies das Versprechen der Übersummativität in sich: Durch die Verschränkung von Interventionsebenen und -formaten erhofft man sich eine potenzierte Wirkung, deren Ausmaß die Summe der Einzeleffekte übersteigt. Systemisch betrachtet ist diese Hoffnung nicht unbegründet, da Anregungen an jedweder Stelle einer Organisation Folgen für das Gesamtsystem nach sich ziehen (Kühl und Muster 2016). Die Kehrseite dieses Effekts liegt in der Gefahr einer Überforderung der Organisation durch mangelnde Beherrschbarkeit. Mehrere Interventionsebenen so miteinander zu verzahnen, dass sie sich gegenseitig befruchten oder zumindest nicht behindern, ist kein einfaches Unterfangen. In der Literatur werden schlecht aufgesetzte Projektpläne als einer der Hauptgründe für das Scheitern solcher Vorhaben aufgeführt (Kunert und von der Weth 2018). Im Folgenden stellen wir acht systemische Prinzipien vor, die es hier zu beachten gilt, um das volle Potenzial einer komplexen Projektarchitektur zur Entfaltung zu bringen.

\section{Prinzipien für Mehrebenen-Ansätze in der Organisationsentwicklung}

\subsection{Spezifität und Symmetrie}

Mehrebenen-basierte Projektarchitekturen erfordern symmetrische, d.h. größenangepasste Elemente. Zugleich benötigen verschiedene Ebenen bzw. Zielgruppen spezifische Formate, um ihren jeweiligen Bedürfnissen gerecht zu werden. Konkret 
bedeutet dies, dass die Verfahren zur Diagnostik bzw. Evaluation, die Interventionen sowie die Projektsteuerung jeweils die Komplexität des Gesamtvorhabens abbilden müssen.

Wenn z.B. die Initiierung einer nachhaltigen Innovationskultur sowohl auf der Personen- als auch auf der Team- und der Organisationsebene ansetzen soll, braucht es zur Ist-Analyse ein Erhebungsverfahren, das diese drei Ebenen ebenfalls adressiert. Es kann sich diesbezüglich noch immer um einen klassischen Fragebogen oder einen Interviewleitfaden handeln, nur sollte der gewonnene Datensatz die erforderliche Komplexität aufweisen, um auf allen Ebenen eine differenzierte Aussage treffen zu können. Hier gilt es, geeignete Erhebungsverfahren (z.B. Kunert 2016) bzw. Handlungsleitfäden (z. B. Kunert 2014) zu nutzen.

Gleiches gilt für die Interventionen. Selbstredend müssen Entwicklungsvorhaben bei Einzelpersonen methodisch anders angegangen werden als auf der Team- bzw. der Organisationsebene. Die Versuchung liegt jedoch nahe, in Mehrebenen-Architekturen mit einer one-size-fits-all-Lösung alles mit demselben Verfahren bzw. mit denselben Beratern zu lösen, um an dieser Stelle Komplexität aus dem Projekt herauszunehmen. Wenn zur Veränderung der Innovationskultur ein IdeenmanagementTool eingeführt wird, dessen Kennzahlen sowohl die einzelne Mitarbeiterin als auch ihre Abteilung sowie das gesamte Unternehmen dazu animieren soll, Neuerungen gegenüber offener zu sein, ist die Gefahr groß, dass der erhoffte Effekt ausbleibt (Bedenk und Popp 2017). Dies kann daher rühren, dass auf den verschiedenen Ebenen völlig unterschiedliche Gründe dafür verantwortlich sind, wieso keine Innovationen angestoßen werden.

$\mathrm{Zu}$ guter Letzt muss auch das begleitende Projektmanagement auf die Komplexität des Vorhabens bezogen sein. Dies gilt für die Administration, die Projektsteuerung bis hin zur Projektkommunikation. Mit zunehmender Anzahl und Spezialisierung der Beteiligten steigt vor allem der Koordinierungsbedarf. Es müssen angemessene Formate gefunden werden, um den Informationsfluss wie auch die Entscheidungsgüte auf hohem Niveau zu halten. So gewinnt die Klaviatur der klassischen Projektgremien - von der Projektsteuerungsgruppe über Lenkungskreis und Resonanzgruppe bis hin zu Staff-Meetings - an enormer Bedeutung (Piber 2020).

\subsection{Oszillation}

In komplexen Organisationsentwicklungsvorhaben ist es enorm wichtig, dass sich die Institution und das Projekt bzw. die Betroffenengruppen untereinander von Zeit zu Zeit gegenseitig darüber unterrichten, welchen Veränderungsprozess sie jeweils gerade durchleben und worauf sie zusteuern. Im besten Fall wirkt dies befruchtend füreinander, im schlimmsten Fall drohen sich die separierten Dynamiken aus den jeweiligen Interventionen zu stören oder gar gegenläufig zu sein. In der Projektarchitektur sollten sich daher divergente Formate der Themenöffnung, Tiefung und Erarbeitung mit konvergenten Methoden, die der Vergemeinschaftung, der Rückmeldung und dem Schließen dienen, abwechseln, wodurch es zu einer Oszillation kommt.

Dieser pulsierende Charakter verlangt auf der Methodenseite einen Wechsel von der Arbeit in Kleingruppen und in der Großgruppe, von abteilungsspezifischen und 
abteilungsübergreifenden Teilnehmenden-Kreisen, von hierarchiefreien und hierarchie-integrierenden Konstellationen. Darüber hinaus lassen sich auf diese Weise auch ein deduktives Vorgehen im Top-down-Ansatz und ein induktives Prozedere im Graswurzel-Stil (Glasl 2020) miteinander verknüpfen.

\subsection{Integration}

Die größte Gefahr von komplexen Projektarchitekturen lauert in der Separierung einzelner Stränge. Wenn z.B. in einem Projekt zur Führungskultur die Arbeit mit den Vorständen nicht mit der Intervention im mittleren und im unteren Management gekoppelt ist, droht sie sich abzuspalten und unterminiert die potenzierte Wirkung. Die Verzahnung sollte in allen Teilsystemen einer Organisationsentwicklung erfolgen.

Im Klientensystem lässt sie sich durch personelle Überschneidungen erreichen. So können Gremien bzw. Formate mit denselben Personen mehrfach besetzt werden, wodurch die Verknüpfung von Interventionssträngen intraindividuell erfolgt. Analog dazu lässt sich eine thematische Überschneidung durch die Bearbeitung derselben Themen in verschiedenen Formaten bzw. Personenkonstellationen erreichen.

Diese Art der Überlappung kann zudem auf die Unterstützungssysteme übertragen werden. Im Projektteam sind Tandems aus Organisationsentwicklern bzw. aus Führungskräften und Berater/innen, die in unterschiedlichen Konstellationen und wechselnden Verantwortlichkeiten miteinander kooperieren, eine gute Option. Hinzukommen vergemeinschaftende Formate wie Projektsteuerungsmeetings, Klausurtage und Supervision. Unterstützt wird die Integration zudem durch ein angemessen komplexes Ablage- und Dokumentationssystem.

\subsection{Choreographie}

Organisationsentwicklungsprozesse durchlaufen unterschiedliche Phasen. Jede ist gekennzeichnet durch besondere Bedürfnisse und Notwendigkeiten, die durch die Qualität und Intensität der Beratungsformate berücksichtigt werden sollten.

$\mathrm{Zu}$ Beginn eines Organisationsentwicklungsprozesses dominieren sehr grundsätzliche Fragen. Thema, Weg und Ziel der Organisationsentwicklung müssen gefunden, verifiziert und kommuniziert werden. Dieser Prozess des Sich-Einspielens in Thema (Was wird bearbeitet?), Ton (Wie wird gearbeitet?), Takt (In welcher Geschwindigkeit wird gelernt und verändert?) und Gestalt (Welches Format hilft dabei?) wird in der Architektur durch eine anfänglich hohe Intensität entsprechend berücksichtigt, um genügend akzelerierende Kräfte freizusetzen (Lewin 1947). Das verlangt ein hohes zeitliches Commitment, was häufig auch ein wertvoller Lackmustest für die Konsequenz ist, mit der ein Veränderungsvorhaben angegangen werden soll (Berenbold und Vögel 2021).

Begleitet eine Consultingfirma diesen Prozess, stellt sich zudem die Frage, welche Rolle die Beratung in diesem Prozess einnimmt, welche Verantwortung, Rechte, Pflichten sie hat und wie sie mit den Mitarbeiter/innen der Institution in Beziehung steht. Organisation und Berater/innen bilden ein neues gemeinsames Beratungssystem, das sich innerhalb kurzer Zeit einspielen muss (Königswieser und Exner 2019). 
Dessen Konstituierung ist eine Herausforderung: Einerseits müssen sich die Protagonisten lernend aufeinander einspielen, d.h. auch wechselseitiges Nicht-Wissen, Verhandlungen der Rollen, sogar das Scheitern von Interventionen gemeinsam erleben. Andererseits sind Beratungssysteme auf Anfangserfolge angewiesen, um die Motivation zu Beginn eines Veränderungsprozesses für sich zu nutzen.

Die Intensität der beraterischen Begleitung und somit die Frequenz an Formaten können dann im Laufe des Organisationsentwicklungsprozesses sukzessive abnehmen, und gegen Ende des Veränderungsprojekts kann die beraterische Unterstützung zunehmend auslaufen. Mehr und mehr wird die Prozessgestaltung wieder den organisationalen Akteuren übergeben. Die Beratungsfirma verabschiedet sich allmählich wieder aus der Organisation. Hier spiegelt sich auf Architekturebene ein Prinzip, das auch aus der Choreographie singulärer Beratungsdienstleistungen bekannt ist: So gibt es im Laufe eines Coachingprozesses oft auch zu Beginn der Zusammenarbeit eine höhere Dichte an Sitzungen, die mit der Dauer sukzessive abnehmen. Ein wichtiges Merkmal professioneller Beratungsarbeit ist hier wie da: Der Klient soll in seinem Lern- und Entwicklungsprozess beraterisch begleitet, keinesfalls jedoch von dieser Unterstützungsfunktion abhängig gemacht werden (Lackner 2016).

\subsection{Phasen}

Erfolgreiche Veränderungen haben eine lange Anlaufphase und eine oft noch längere Habituierungsphase. Darauf wies schon Lewin (1947) in seinem berühmten Modell der Veränderung hin. Was wir gemeinhin als Change bezeichnen, nämlich die mehr oder weniger sichtbare Veränderung eines Verhaltens, findet nicht schlagartig statt. Sie ist eingebettet in eine Vorbereitungsphase, die die Bereitschaft und Fähigkeit zur Veränderung erst ermöglicht (Unfreeze), und in eine Phase der Nachhaltigkeitssicherung (Refreeze). Einen solchen Dreischritt aus Vorbereitung, Veränderung und Nachbereitung benötigen Personen wie Organisationen, wenngleich er keineswegs sequentiell und einmalig ablaufen muss, sondern vielmehr Ausdruck rivalisierender sozialer Kräfte ist (vgl. Cummings et al. 2016). Entsprechend bilden nachhaltige Beratungsarchitekturen diesen Dreischritt ab.

Bevor es an „des Pudels Kern“der Veränderung geht, müssen in einem Beratungsprozess die Motivatoren und Kompetenzen zur Veränderung erst erhoben werden. So geht einem organisationalen Veränderungswunsch (z. B. nach einer neuen Innovationskultur) notwendigerweise eine Phase der intrinsischen Tiefung dieses Wunsches voraus: Warum soll ein altes (organisationales) Muster aufgegeben werden? Wie kam es zu diesem Muster, welchen Nutzen und welche Kosten hatte es? Was sind Nutzen und Kosten des neuen (gewünschten) Musters? Was ist jeder Einzelne bereit, für einen neuen Umgang mit Ideen aufzugeben? Letztlich geht es in der Unfreeze-Phase um eine ehrliche Einschätzung, wie ernst der Veränderungswunsch allen Beteiligten ist (vgl. Bedenk 2014). Gute Projektarchitekturen ermöglichen Räume, in denen die Protagonisten sich diesen Fragen ehrlich stellen und sie beantworten können. Im Idealfall passiert dies multimodal, etwa durch Einzelgespräche (Coaching, Interviews), Gruppenformate (Workshops, moderierte Teambesprechungen) oder anonyme Erhebungen (Fragebogen-Formate). 
In gleicher Weise berücksichtigen Projektpläne die Notwendigkeit zur Einübung, Gewöhnung und Verstetigung eines veränderten Verhaltens. Der Rückfall in alte Verhaltensmuster ist ein Phänomen, das Organisationen oft erleben (Kunert und Staar 2018). Ein neues Verhalten wurde zwar kennengelernt (Change), aber nicht ausreichend verinnerlicht, oder dessen Akzeptanz ist in den alten Konstellationen nur schwer erreichbar. Entwicklungsarchitekturen widmen sich daher der Frage, wie sich ein gewünschter Habitus gut in den Arbeitsalltag transferieren lässt. Dies beginnt mit der Einübung über einen längeren Zeitraum hinweg, sodass sich ein neues Verhalten auch als neues Muster durchsetzen kann. Beratung kann den Prozess der Einübung dabei durch Feedback oder Feedback-Formate unterstützen. Schließlich geht es um Verstetigung: Das neue Verhalten wird zunehmend zum Gewohnten ,eingefroren“ und somit zu einer neuen Kompetenz, die unter der Bewusstseinsschwelle wirksam wird.

\subsection{Kaskadierung}

Eine häufige Einstiegsfrage bei komplexen Wandelprozessen lautet: Wo fängt man an? Glasl (2020) gibt darauf eine strategische Antwort und unterscheidet Top-downund Bottom-up-Ansätze sowie die Mischformen bi-polar, Keil und Ölfleck. Der Reiz bei komplexen Projektarchitekturen liegt darin, Mischstrategien verfolgen zu können. So lassen sich unter der Überschrift einer neuen Innovationskultur ein Leitbildprozess vom Management her, die Entwicklung eines Ideenmanagements aus der Führungsebene sowie ein Organisationsklima-Prozess aus der Belegschaft heraus simultan initiieren. Wichtig ist nur, dass die jeweilige Strategie ihre Entsprechung in der Architektur findet, indem sowohl die notwendigen Formate angelegt als auch die Integration gesichert werden.

\subsection{Agilität und Adaptivität}

Jede Architektur ist eine Antizipation des Weges, den eine Organisation im Laufe ihrer Entwicklung gehen wird. Sie ist die Landkarte für ein Gebiet, das Organisation und Berater/innen noch nicht kennen und erst durch das „Tun“ kennenlernen. Insofern gilt für Architekturen, was für alle Modelle gilt: Sie sind notwendigerweise nur eine Hypothese und können bestenfalls richtungsweisend sein (Königswieser und Exner 2019). Sie liefern Vorschläge, wie die nächsten Schritte in die unbekannte Landschaft tastend vorgenommen werden können. In diesem Sinne sind sie nicht in Stein gemeißelt, sondern agil. Sie verändern sich mit jeder neuen Erfahrung, die Klienten- und Beratersystem gemeinsam machen (Schiersmann 2009).

Vor allem aber sind Architekturen adaptiv. Der Begriff „Adaptivität“ weist auf die evolutionäre Natur von Entwicklungsprozessen hin. Am Anfang eines Organisationsentwicklungsprozesses stehen Momente der Variation (Kühl und Muster 2016). Die Organisation probiert für sich neue Verhaltensweisen aus, die Berater/innen testen neue Interventionen, und im co-kreativen Zusammenspiel variieren beide ihre Interaktionen. Dieser öffnende und überproduktive Prozess ist notwendig, da er erst den Optionsraum für eine Selektion ermöglicht: Welcher dieser Versuche ist adaptiv 
für die Organisation und bringt sie aktuell einen Schritt weiter? Adaptives Vorgehen ist somit ein schrittweises und relatives Agieren.

Für Organisationsentwickler bedeutet adaptive Arbeit zunächst, dass jede Architektur ein mentales Modell darstellt, das für den Moment Handlungsfähigkeit herstellt. Da sich die Momentaufnahmen permanent aktualisieren, muss analog auch die Planung aktualisiert werden. Für Berater/innen heißt dies, die Architektur regelmäßig neu zu studieren, sie zu hinterfragen und anzupassen.

Die Adaptivität von Architekturen ist auch ein elementarer Bestandteil der Klientenprofessionalisierung, denn viele Klienten haben den verständlichen Wunsch nach einem Projektplan, nach einer Entwicklungsagenda oder einer Roadmap. Berater/ innen können diesen Wunsch nach Orientierung mit einer Architektur (teilweise) erfüllen; jede Architektur zeichnet sich in ihrer Professionalität jedoch paradoxerweise gerade dadurch aus, dass sie kritisch hinterfragt bleibt und im Laufe des Prozesses ihr Aussehen ändern wird.

\subsection{Anschlussfähigkeit und Irritation}

Jede systemische Beratungsarbeit findet in einem sensiblen Spannungsfeld zwischen Anschlussfähigkeit und Irritation statt. Anschlussfähigkeit beschreibt die Fähigkeit des Beratersystems, die Gepflogenheiten, Gewohnheiten, Verhaltens- und Redeweisen, sprich die Kommunikationen und Kommunikationsmuster eines Systems hinreichend zu verstehen, um sie auch selbst adressieren zu können. Beratung kann erst dann funktionieren, wenn sie in einer Organisation gehört wird und nicht im Rauschen landet.

Versteht man Organisationsentwicklung als Weg hin zu etwas Neuem, darf Beratung verständlicherweise nicht nur die Berücksichtigung von Bekanntem im Blick haben. Sie liefert ebenso Impulse für Veränderung und irritiert, etwa durch den Einsatz vorher unbekannter Formate. Im Beratungsprozess lernen Mitarbeiter/innen einer Organisation sich (z.B. im Coaching), ihr Team (z.B. in Teamworkshops) oder ihre Organisation (z. B. in der Großgruppe) neu kennen. Ein wichtiges Element guter Architekturarbeit ist dabei die abgestimmte Balancierung von Anschlussfähigkeit und Irritation über den Prozess hinweg. So sollte es einzelne Projektelemente geben, die tendenziell eher das Gewohnte bedienen, um Sicherheit und Vertrauen zu stärken, sowie andere Formate, die der Irritation in all ihren produktiven Formen dienen, ohne jedoch die Organisation zu überfordern.

\section{Praxisbeispiel}

Im Folgenden wollen wir diese Prinzipien an einem Fallbeispiel exemplarisch darstellen. Es handelt sich um eine derzeit erfolgende Organisationsentwicklung in einem Kunst- und Museumsbetrieb, die auf 18 Monate angelegt ist. Das Organigramm umfasst eine Doppelspitze als Vorstand, 6 Abteilungsleiter/innen und insgesamt 80 Beschäftigte. Ziel ist es, eine konstruktive Kommunikationskultur im Haus wiederherzustellen, Vertrauen zueinander zurückzugewinnen und die Kooperation zwischen den Abteilungen wie auch den Hierarchieebenen zu stärken. 

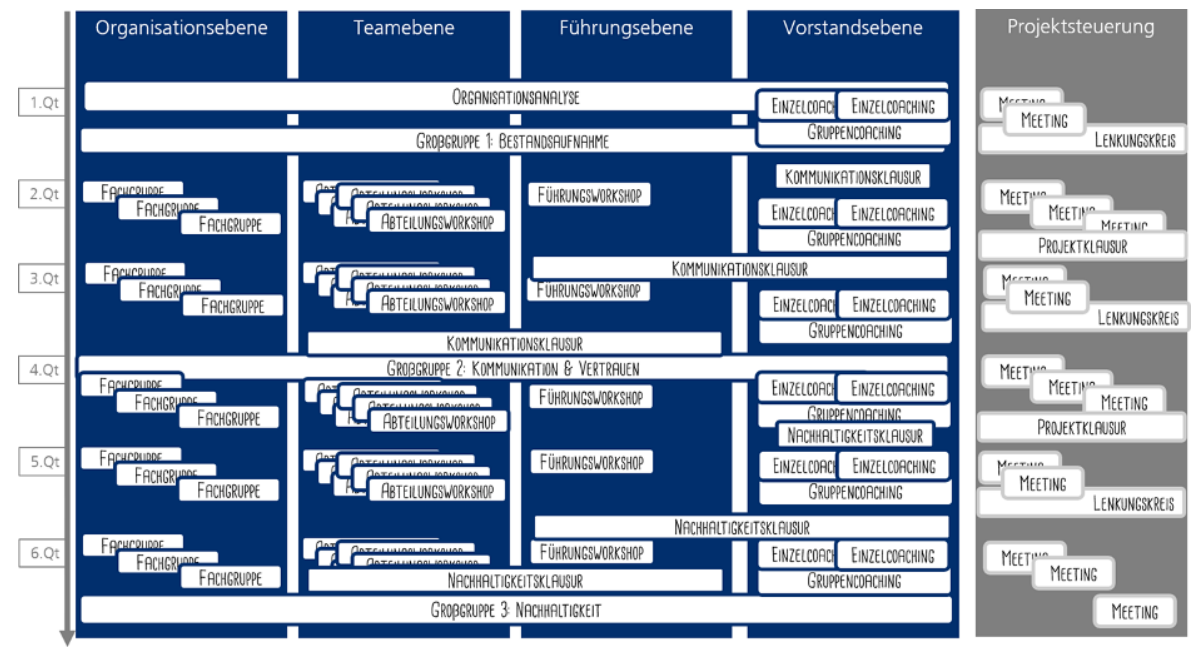

Abb. 1 Mehrebenen-Architektur in einem Kunst- und Museumsbetrieb mit 4 Interventionsebenen

Die Planung folgte einer Mehrebenen-Architektur mit den 4 Säulen Vorstand, Führungskräfte, Teams und Gesamtorganisation. Sie ist in Abb. 1 dargestellt. Die Formate reichen von Einzelcoachings über Workshops und Schulungen bis hin zu Großgruppenveranstaltungen. Das Projektmanagement übernimmt eine paritätisch besetzte Steuerungsgruppe bestehend aus vier Mitarbeiter/innen und vier Berater/ innen.

Um dem Prinzip der Spezifität und Symmetrie gerecht zu werden, hat jede Interventionsebene ihr eigenes Format. Die beiden Vorstände arbeiten pro Quartal sowohl im Einzelsetting als auch im Tandem mit einem Coach. Die Abteilungsleiter/innen reflektieren ihre Führungsrolle in einem regelmäßigen Workshop. Ebenso bearbeiten die Abteilungen ihre spezifischen Themen in eigenen moderierten Veranstaltungen. Auf Organisationsebene wurden sogenannte Fachgruppen ins Leben gerufen, die Veränderungsthematiken der gesamten Institution versorgen. An Intensität und beraterischer Versorgung gleichen sich die Formate, womit gewährleistet wird, dass jede Interventionsebene ähnlich große Aufmerksamkeit erhält. Zugleich wird sichergestellt, dass Veränderungsvorhaben zielgruppenspezifisch und tiefend (divergent) angegangen werden können. In der Projektsteuerung sind die Meetings das entsprechende Äquivalent. Dialektisch dazu dienen die Großgruppenveranstaltungen bzw. der Lenkungskreis als konvergente Formate dem gegenseitigen Unterrichten und Schließen von Themen.

Als drittes Element wurde eine Reihe von Klausuren in die Architektur aufgenommen. Hier werden kaskadisch - beginnend mit einer Vorstandsklausur und endend mit Teamklausuren - Themen top-down erarbeitet und weitergegeben. Die Fachgruppen wiederum sind das bottom-up-Pendant dazu. Beide Stränge treffen sich wiederum in der Großgruppe. Durch die Wiederholung dieses Vorgangs erzeugt das Projekt eine Oszillation von divergenten und konvergenten Formaten sowie die Verzahnung zweier kaskadierender Prozesse. 
Die Integration wird zum einen thematisch erzeugt, wenn sowohl der Vorstand allein als auch mit den Führungskräften und wiederum diese mit ihren Abteilungen an demselben Thema arbeiten. Zum anderen sichert die Projektgruppe durch ihre monatlichen Meetings, halbjährlichen Klausuren und ein geteiltes Ablage- und Dokumentationssystem die Verzahnung in der Projektsteuerung.

Die Unfreeze-Phase ist in der Architektur durch die Organisationsanalyse sowie die erste Großgruppe realisiert. In letzterer nehmen die Mitarbeiter/innen Tuchfühlung mit dem Projekt (und den Berater/innen) auf, erproben die Selbstreflexion und heben Themen. Das Refreezing wird im vorliegenden Fall vor allem thematisch angegangen, indem das Thema Nachhaltigkeit im Sinne einer Rückfallprävention und der Institutionalisierung konstruktiver Kommunikationsformen im Mittelpunkt steht.

$\mathrm{Zu}$ guter Letzt sei noch erwähnt, dass es sich bei die Architektur in Abb. $1 \mathrm{um}$ eine temporäre Planung handelt, die im Sinne der Agilität und Adaptivität bei jedem Steuerungsgruppen-Meeting hinterfragt und nicht selten geändert wird.

\section{Fazit}

Mit diesem Artikel wollen wir für die Bedeutung von Projektarchitekturen in der Beratung komplexer Organisationsentwicklungen sensibilisieren. Begriff und Prinzip der Architektur sind aus dem klassischen Projektmanagement wohl bekannt. Gleichzeitig regt der Transfer in die systemische Organisationsberatung zu weitergehenden Überlegungen an. In unserem Beitrag beleuchten wir vorrangig die Chancen, die eine Architekturarbeit zum einen innerhalb einzelner Formatgestaltungen und zum anderen in der Synergie zwischen diesen bietet.

Architekturen ermöglichen es zunächst, innerhalb professioneller Interaktionsformate die Potenziale systemischer Arbeit im großen Maße zu entfalten. Die wiederkehrende Arbeit mit Einzelpersonen (z.B. im Coaching), Gruppen (z. B. in Workshops) und ganzen Organisationen (z. B. in Großgruppen) wird dramaturgisch vorausgedacht. Wir beschreiben die Möglichkeit choreographierter (3.4) und phasisch (3.5) angelegter Beratungsprozesse, ohne sich dabei die Option auf Adaptivität und Agilität (3.7) zu nehmen. Die Qualität solcher Projektpläne erlaubt es Berater/innen und Klient/innen, planerisch gesichert und inhaltlich unabhängig Zeit und Raum zur Verfügung zu stellen, um die Qualitäten systemischer Arbeit voll zur Geltung zu bringen (vgl. Königswieser und Exner 2019, S. 47). So werden Wechselspiele aus Reflexion und Aktion, Neues lernen und Wissen anwenden, Feedback geben und es nehmen, Traditionen erkennen und sie verändern in vielfacher Weise möglich.

Zum anderen erlauben komplexe Architekturen das integrierte Verzahnen einzelner Interventionen über mehrere Interventionsebenen hinweg (vgl. das obige Praxisbeispiel). In unseren Augen ist diese beraterische Arbeit gerade in langwierigen Organisationsentwicklungsprojekten von besonderer Bedeutung, da hier verschiedene Formate parallel existieren bzw. sequentiell aufeinander abgestimmt durchgeführt werden können. Dies beginnt schon in der Analysephase (3.1 Spezifität und Symmetrie) und setzt sich fort in der nachfolgenden Arbeit mit der Organisation. Wir diskutieren Möglichkeiten der Integration und der Verzahnung (3.3), um Spaltungseffekte durch divergierende Impulse zu vermeiden. Zudem verweisen wir auf die 
besondere Chance bei Mehrebenen-Ansätzen, dass Klient/innen wichtige Organisationsthemen in festen sowie immer wieder neuen Personenkonstellationen bearbeiten können. Ein Wechsel aus öffnenden und schließenden, kreativen und fokussierenden Prozessen wird auf diese Weise möglich (3.2 Oszillation). Dafür braucht es das gesamte Ensemble beraterischer Formate, die analog zu einem Orchester aufeinander abgestimmt interagieren. Das Dirigieren dieses Systems geschieht im Wesentlichen durch die Komposition komplexer Projektpläne, die durch einen Mehrebenen-Ansatz die verschiedenen Veränderungsebenen Person, Team und Organisation passgenau adressieren und zugleich miteinander verbinden.

Funding Open Access funding enabled and organized by Projekt DEAL.

Open Access Dieser Artikel wird unter der Creative Commons Namensnennung 4.0 International Lizenz veröffentlicht, welche die Nutzung, Vervielfältigung, Bearbeitung, Verbreitung und Wiedergabe in jeglichem Medium und Format erlaubt, sofern Sie den/die ursprünglichen Autor(en) und die Quelle ordnungsgemäß nennen, einen Link zur Creative Commons Lizenz beifügen und angeben, ob Änderungen vorgenommen wurden.

Die in diesem Artikel enthaltenen Bilder und sonstiges Drittmaterial unterliegen ebenfalls der genannten Creative Commons Lizenz, sofern sich aus der Abbildungslegende nichts anderes ergibt. Sofern das betreffende Material nicht unter der genannten Creative Commons Lizenz steht und die betreffende Handlung nicht nach gesetzlichen Vorschriften erlaubt ist, ist für die oben aufgeführten Weiterverwendungen des Materials die Einwilligung des jeweiligen Rechteinhabers einzuholen.

Weitere Details zur Lizenz entnehmen Sie bitte der Lizenzinformation auf http://creativecommons.org/ licenses/by/4.0/deed.de.

\section{Literatur}

v. Ameln, F., \& Kramer, J. (2007). Organisationen in Bewegung bringen - Handlungsorientierte Methoden für die Personal-, Team- und Organisationsentwicklung. Heidelberg: Springer.

Ashby, W. R. (1956). An introduction to cybernetics. New York: Wiley.

Bedenk, S. (2014). Change story workshops. In J. Hüttner \& R. Träder (Hrsg.), Toolbox - Schrittmacher für Innovationen (S. 108-113). Berlin: artop.

Bedenk, S., \& Popp, M. (2017). Das Ideenmanagement auf der Suche nach Problemlösungen. Wissensmanagement, 1, 36-37.

Berenbold, S., \& Vögel, S. (2021). Wirksamer Change - ein Appell gegen halbherzige Veränderungsvorhaben. Organisationsentwicklung, 21(1), 44-47.

Cummings, S., Bridgman, T., \& Brown, K.G. (2016). Unfreezing change as three steps: rethinking Kurt Lewin's legacy for change management. Human Relations, 69(1), 33-60.

Glasl, F. (2020). Strategien der Organisationsentwicklung. In F. Glasl, T. Kalcher \& H. Piber (Hrsg.), Professionelle Prozessberatung. Das Trigon-Modell der sieben OE-Basisprozesse (S. 429-470). Bern: Haupt.

Heitger, B., \& Serfass, A. (2015). Unternehmensentwicklung - Wissen, Wege, Werkzeuge für morgen. Stuttgart: Schäffer-Poeschel.

Königswieser, R., \& Exner, A. (2019). Systemische Intervention - Architekturen und Designs für Berater und Veränderungsmanager (9. Aufl.). Stuttgart: Schäffer-Poeschel.

Kranz, O. (2009). Interaktion und Organisationsberatung: Interaktionstheoretische Beiträge zu Profession, Organisation und Beratung. Wiesbaden: VS.

Kühl, S., \& Muster, J. (2016). Organisationen gestalten. Wiesbaden: Springer VS.

Kunert, S. \& Bedenk, S. (2013). Die Professionalisierung der Organisationsberatung und die Rolle der Wissenschaft. Organisationsberatung, Supervision, Coaching, 20(1), S. 17-22.

Kunert, S. (2014). Innovationsprojekt-Analyse IPA. In J. Hüttner \& R. Träder (Hrsg.), Toolbox Schrittmacher für Innovationen (S. 100-107). Berlin: artop. 
Kunert, S. (2016). Kultur fördert Innovationen: Organisationsdiagnostik in Zeiten von New Work. Wirtschaftspsychologie aktuell, 23(2), 55-57.

Kunert, S., \& Staar, H. (2018). Failure in organizational change. In S. Kunert (Hrsg.), Strategies in failure management - scientific insights, case studies and tools (S. 9-25). Heidelberg: Springer.

Kunert, S., \& von der Weth, R. (2018). Failure of projects. In S. Kunert (Hrsg.), Strategies in failure management-scientific insights, case studies and tools (S. 47-66). Heidelberg: Springer.

Lackner, K. (2016). Scheitern in der Beratung. In S. Kunert (Hrsg.), Failure Management -Ursachen und Folgen des Scheiterns (S. 133-149). Wiesbaden: Springer.

Lewin, K. (1947). Frontiers in Group Dynamics. Human Relations, 1(2):143-153.

Lippold, D. (2020). Grundlagen der Unternehmensberatung. Berlin: De Gruyter Oldenbourg.

Piber, H. (2020). Change-Management-Prozesse. In F. Glasl, T. Kalcher \& H. Piber (Hrsg.), Professionelle Prozessberatung. Das Trigon-Modell der sieben OE-Basisprozesse (S. 375-427). Bern: Haupt.

Schanz, G. (1994). Organisationsgestaltung. Management von Arbeitsteilung und Koordination (2. Aufl.). München: Vahlen.

Schiersmann, C. (2009). Selbstorganisation und Problemlösen als Eckpunkte einer allgemeinen Beratungstheorie. In H. Pühl (Hrsg.), Handbuch Supervision und Organisationsentwicklung (S. 103-122). Wiesbaden: VS.

Schlick, C.M., Bruder, R., \& Luczak, H. (2018). Arbeitswissenschaft (4. Aufl.). Wiesbaden: Springer Vieweg.

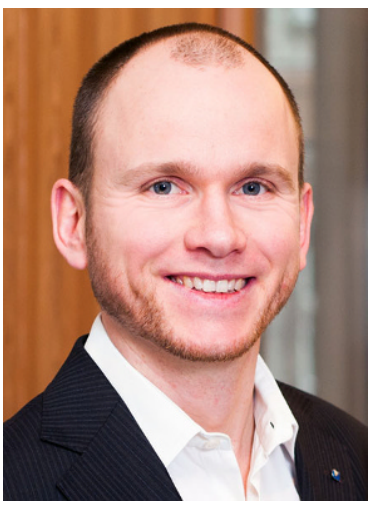

Dr. S. Kunert ist Dipl.-Psych., systemischer Organisationsberater, Gestalt-Coach, Ausbilder und Hochschuldozent. Er ist Koordinator des Masterstudiengangs Beratung und Beratungswissenschaft an der Humboldt-Universität zu Berlin. Seine Forschungsschwerpunkte liegen in den Bereichen Organisation, Kultur, Change Management, Führung sowie Scheitern.

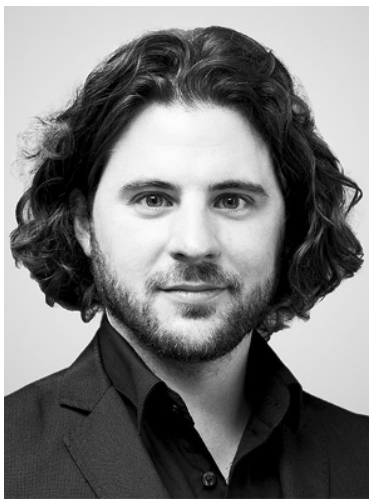

Dr. S. Bedenk ist Dipl.-Psych., systemischer Organisationsberater, systemischer Coach und Trainer bei artop - Institut an der HU Berlin. Er ist Teil des Ausbilder/innenteams der ,systemischen Organisationsberatung" bei artop. Seine Forschungsschwerpunkte liegen in den Bereichen individuelle und organisationale Entscheidungsfindung, strategisches Management und Innovation. 